\title{
A produção científica da Odontologia e a Agenda Nacional de Prioridades de Pesquisa em Saúde
}

\author{
Doris Gomes*; Igor Greik Agnoletto*; Marina Leite Souza*; Vinícius Spiger*; João Rodolfo Gomes \\ Jakymiu*; Elisa da Cunha Fujii**; Ana Lúcia Schaefer Ferreira de Mello***
* Pós-graduando na área de Odontologia em Saúde Coletiva, Universidade Federal de Santa Catarina
Cirurgiã-dentista, Prefeitura Municipal de Joinville
Docente do Departamento de Odontologia e do Programa de Pós-Graduação em Odontologia, área Odontologia em Saúde \\ Coletiva, Universidade Federal de Santa Catarina
}

Recebido em 15/01/2017. Aprovado em 16/04/2017.

\begin{abstract}
RESUMO
O estudo analisou a produção atual do conhecimento científico em saúde bucal nos cursos de pósgraduação em Odontologia (stricto-sensu) sul-brasileiros, relacionando-a às diretrizes traçadas pela Agenda Nacional de Prioridades de Pesquisa em Saúde (ANPPS). Trata-se de uma pesquisa de abordagem quanti-qualitativa, de natureza exploratório-descritiva a partir de análise documental. Os dados, coletados de sítios eletrônicos oficiais no primeiro semestre de 2015 , consistem em teses e dissertações produzidas entre 2011 e 2015, pelos programas de pós-graduação stricto-sensu em "Odontologia" ou "Ciências Odontológicas", de Universidades Federais do Sul do país. Após leitura dos títulos e resumos, procedeu-se a classificação quanto à aderência das produções aos eixos da ANPPS, com posterior organização, tabulação, análise quantitativo-descritiva e análise qualitativa, discutindose possíveis lapsos entre políticas públicas e práticas, na implementação da ANPPS. A maior parte da produção de teses e dissertações analisadas não apresentou aderência aos tópicos da ANPPS $(56,13 \%)$ relacionados à saúde bucal, sendo que as áreas de Odontopediatria e Saúde Coletiva detiveram as maiores médias de produção vinculadas (40,8\%). Embora existam movimentos de assimilação e incorporação à ANPPS na pesquisa em saúde bucal, disciplinas clínicas tradicionais ainda não referenciam à Agenda e sua prioridade em estudos voltados às necessidades populacionais e interesses do Sistema de Saúde. A incorporação da ANPPS à pesquisa odontológica pode transformar a Odontologia brasileira em nova referência de construção de conhecimento para além do contexto nacional.
\end{abstract}

Descritores: Saúde Bucal. Odontologia. Educação de Pós-graduação em Odontologia.

\section{INTRODUÇÃO}

Muitos são os fatores que impulsionaram avanços no nível do profissionalismo da Odontologia brasileira nas últimas décadas, relacionados ao crescente investimento em formação associado à pesquisa na área da Odontologia; aumento significativo de cursos de graduação, lato $e$ stricto sensu; importante adesão de uma legião de novos profissionais às entidades representativas de classe; fortalecimento técnico do ensino universitário público seguido pela busca de novos conhecimentos no exterior bancados por órgãos 
financiadores estatais; multiplicação de eventos científicos da área; e edição de novas revistas e periódicos científicos ${ }^{1,2}$.

Desta maneira, a Odontologia brasileira foi alçada a um lugar de destaque no cenário internacional, representando cerca de $7 \%$ de toda a produção científica nos últimos anos ${ }^{3,4}$. Entre 1996 e 2015, as 16.333 publicações contabilizadas elevaram o Brasil ao patamar de segundo país com maior número de publicações científicas, superado apenas pelos Estados Unidos da América $^{5}$ - uma diferença que vem diminuindo a cada ano $^{6}$. Um fenômeno considerado recente, iniciado neste século, quando a Odontologia brasileira apresenta uma das maiores médias de crescimento no número de publicações científicas ${ }^{3}$.

Entretanto, o Fator H do Brasil é apenas o $4^{\circ}$ maior na área e as citações são consideradas de baixo impacto (média de 6,3 citações), o que remete o país ao $12^{\circ}$ lugar no cenário internacio$\mathrm{nal}^{5}$. Além disto, grande parte dessas publicações acontece em revistas Qualis B, segundo classificação da Coordenação de Aperfeiçoamento de Pessoal de Nível Superior (CAPES), sendo majoritariamente quantitativas, o que não representa, necessariamente, acréscimo de qualidade ${ }^{7}$. Muitos dos temas de recorrente interesse das pesquisas nas ciências odontológicas, não se mostram capazes de reduzir as doenças e o sofrimento de maneira ampla e sustentável à sociedade brasileira ${ }^{8}$. O resultado da Pesquisa Nacional de Saúde Bucal (SB Brasil 2010) ${ }^{9}$, permite identificar que as doenças bucais ainda têm um impacto negativo na população, imperando as iniquidades no acesso aos serviços odontológicos e nas condições de saúde. Neste sentido, a pesquisa em saúde bucal (SB) deveria investir maior esforço, por meio da adoção de direcionamentos estratégicos, em evitar atividades de baixo valor científico e pouco impacto social ${ }^{10}$.

Contudo, ainda prevalece na Odontologia moderna a articulação entre pesquisa, produção industrial e sistemas de atenção à saúde concebida no modelo Flexneriano de ensino, pautada em progressiva capitalização dos três setores, dando forma ao "complexo médico-industrial". Concretiza-se a produção do conhecimento e o desenvolvimento de pesquisas sobre tecnologias com perfil mercadológico ${ }^{11}$. Por meio de complicados mecanismos burocráticos de patenteamento de novos processos e produtos, sob domínio de grandes empresas transnacionais, verificase quase total ausência de investimentos na pesquisa revertidos para o desenvolvimento da indústria nacional e em benefícios científicos voltados a toda sociedade. Um modelo que convertido em medicalização, passa a justificar o direito à saúde como direito à assistência médica ou odontológica individual e especializada ${ }^{12}$.

Em um caminho divergente desta medicalização, a inserção de Equipes de SB na Estratégia de Saúde da Família já ao final da década de 1990 associada às diretrizes da Política Nacional de Saúde Bucal (PNSB) ${ }^{13}$, lançadas em 2004, amplia consideravelmente o investimento de recursos públicos na área, com o desenvolvimento científico e tecnológico passando a ser considerado um componente estratégico e essencial na concretização da reforma sanitária. Assim, as pesquisas em SB assumem um papel de destaque para construção de uma odontologia democratizada e voltada às necessidades populacionais.

Neste caminho, a Agenda Nacional de Prioridades de Pesquisa em Saúde (ANPPS) ${ }^{14}$ foi construída como um instrumento da Política Nacional de Ciência, Tecnologia e Inovação em Saúde, para viabilizar maior alinhamento entre as prioridades de pesquisa em saúde e os princípios que regem o Sistema Único de Saúde (SUS). Após um diagnóstico inicial da situação de saúde e das condições de vida do país, e com base no debate da $2^{\mathrm{a}}$ Conferência Nacional de Ciência, Tecnologia e Inovação em Saúde, a ANPPS definiu diversas subagendas de pesquisa, onde 
situam-se os 17 tópicos prioritários da subagenda da SB - os quais podem constar de qualquer etapa da cadeia do conhecimento, da pesquisa básica até a operacional, sem restrições quanto às áreas do conhecimento envolvidas e, em muitos casos, associados às prioridades de saúde.

Assim, este estudo objetivou analisar a produção atual do conhecimento científico em SB nos cursos de pós-graduação em odontologia stricto-sensu Sul-brasileiros, relacionando-a às diretrizes traçadas pela ANPPS.

\section{METODOLOGIA}

O presente estudo de abordagem quantiqualitativa, de natureza exploratório-descritiva, do tipo documental, pautou-se na coleta de dados no primeiro semestre de 2015 - teses e dissertações produzidas dos anos de 2011 a 2015 -, nos sítios eletrônicos dos programas de Pósgraduação stricto-sensu em "Odontologia" ou "Ciências Odontológicas", de 5 Universidades Federais da região Sul do Brasil: Universidade Federal do Paraná (UFPR), Universidade Federal de Santa Maria (UFSM), Universidade Federal do Rio Grande do Sul (UFRGS), Universidade Federal de Pelotas (UFPel) e Universidade Federal de Santa Catarina (UFSC).

Os títulos e resumos foram analisados segundo sua aderência aos 17 eixos do capítulo SB da ANPPS (quadro 1). A classificação foi realizada por um grupo de 7 autores, após análise dos títulos e resumos, debatida e fechada em reunião de consenso.

Os títulos considerados aderentes à ANPSS foram inseridos em planilha Excel $^{\circledR}$ compondo um banco de dados com as seguintes variáveis: programa de origem, tipo de texto (tese ou dissertação), ano da defesa e eixo de aderência à agenda. Procedeu-se a análise descritiva destas variáveis.

Em uma segunda etapa, buscou-se uma análise qualitativa pautada na coerência da ANPPS enquanto política pública, articulada aos objetivos estratégicos traçados. Procurou-se compreender a existência de "lapsos" entre políticas e práticas, na implementação da ANPPS ${ }^{15}$.

\footnotetext{
Quadro 1 - Eixos do capítulo saúde bucal da Agenda Nacional de Prioridades de Pesquisa em Saúde

1 Modelos de atenção e serviços de saúde bucal e impacto nos índices epidemiológicos

2 Desenvolvimento de estratégias para promoção de saúde bucal, incluindo ações interdisciplinares, com base nas diretrizes da política nacional de saúde bucal

3 Estudos relativos a fatores de risco e atenção em saúde bucal em grupos vulneráveis e específicos

4 Estudos sobre a prevenção das doenças bucais: utilização de técnicas, produtos e materiais alternativos para prevenção, impacto das ações de programas de higiene bucal e da florestação das águas de abastecimento público

5 Fatores de risco para ocorrência de fissura lábio palatal e de doenças bucais em grupos vulneráveis

6 Estudo do impacto de hábitos alimentares cariogênicos, higiênicos e comportamentais de natureza cultural e socioeconômica da população

7 Estudo sobre causas e consequências da perda dental, edentulismo, câncer bucal e demais patologias bucais

8 Pesquisa sobre os indicadores de saúde bucal

9 Estudos sobre os riscos ocupacionais em profissionais da área odontológica, com ênfase nas hepatites A, B, C, AIDS e exposição ao mercúrio

10 Desenvolvimento e aperfeiçoamento de técnicas e materiais odontológicos, de qualidade e baixo custo, e de equipamentos e produtos portáteis para atendimento em todos os níveis de atenção e em regiões de difícil acesso

11 Estudo sobre etiologia, epidemiologia e impacto da cárie e da doença periodontal na saúde individual e na população

12 Estudo sobre a redução de danos à saúde bucal

13 Estudo dos efeitos da intervenção terapêutica multidisciplinar em respirador bucal

14 Estudo de tratamentos alternativos das doenças bucais de grande demanda populacional

15 Estudo para avaliação da oferta de serviços de saúde bucal

16 Estudo sobre inovação de próteses bucais

17 Desenvolvimento de sistemas de informação de dados epidemiológicos em saúde bucal com livre acesso à população
} 


\section{RESULTADOS}

No período do estudo produziu-se 465 trabalhos, sendo 349 dissertações e 116 teses, com média de 69,8 $\pm 23,43$ dissertações e $23,2 \pm 25,85$ teses, anualmente. Em números absolutos, o quantitativo de dissertações superou em três vezes o de teses defendidas. As instituições detentoras de programas que oferecem mestrado e doutorado (UFPel, UFRGS, UFSC) detêm $71,8 \%$ desta produção.

A maior parte da produção analisada não apresentou aderência a ANPPS (56,13\%). Apesar de concentrarem porção significativa da produção científica total $(59,78 \%)$, UFRGS e UFSC detêm, juntas, a menor proporção média de aderência $(34,67 \%)$ aos tópicos da Agenda e, ainda assim, representam parte substancial do universo total da produção aderida $(47,06 \%)$. UFPel e UFPR possuem a maior proporção média de aderência (71,69\%), com importante representatividade no universo dos resultados (37,26\%). Evidencia-se, a partir destes dados, que maiores quantitativos de produção não significam, necessariamente, maior proporção de aderência à ANPPS (tabela 1).

Constata-se uma média de, aproximadamente, 41 produções científicas relacionadas aos eixos da ANPPS, anualmente, sendo as dissertações responsáveis por $75 \%$ do total de aderências à Agenda. Apesar do predomínio global de adesão das dissertações em relação às teses, as proporções de adesão por tipo são semelhantes $(44,41 \%$ das dissertações e $41,17 \%$ das teses) (tabelas 2 e 3 ).

Tabela 1 - Distribuição das teses e dissertações defendidas nos Programas de Pós-Graduação estudados, entre 2011 e 2015

\begin{tabular}{lccccccc}
\hline & & Defendidas & \multicolumn{5}{c}{ Aderência à ANPPS } \\
& Dissertação & Tese & n & Dissertação & Tese & n & \% \\
\hline UFPel & 38 & 15 & 53 & 23 & 10 & 33 & 62,62 \\
UFPR & 53 & 0 & 53 & 43 & 0 & 43 & 81,13 \\
UFRGS & 82 & 55 & 137 & 34 & 27 & 61 & 44,53 \\
UFSC & 95 & 46 & 141 & 23 & 12 & 35 & 24,82 \\
UFSM & 81 & 0 & 81 & 32 & 0 & 32 & 39,51 \\
Total & 349 & 116 & 465 & 155 & 49 & 204 & 43,87 \\
\hline
\end{tabular}

Tabela 2 - Distribuição das teses e dissertações defendidas nos Programas de Pós-Graduação estudados, segundo ano (2011 a 2015), que tiveram aderência aos eixos da ANPSS

\begin{tabular}{|c|c|c|c|c|c|c|c|c|c|c|c|c|}
\hline & \multicolumn{2}{|c|}{2011} & \multicolumn{2}{|c|}{2012} & \multicolumn{2}{|c|}{2013} & \multicolumn{2}{|c|}{2014} & \multicolumn{2}{|c|}{2015} & \multicolumn{2}{|c|}{ Total } \\
\hline & $\mathbf{n}$ & $\%$ & $\mathbf{n}$ & $\%$ & $\mathbf{n}$ & $\%$ & n & $\%$ & n & $\%$ & $\mathbf{n}$ & $\%$ \\
\hline UFPel & 10 & 4,90 & 14 & 6,86 & 4 & 1,96 & 5 & 2,45 & 0 & 0,00 & 33 & 16,18 \\
\hline UFPR & 11 & 5,39 & 6 & 2,94 & 6 & 2,94 & 9 & 4,41 & 11 & 5,39 & 43 & 21,08 \\
\hline UFRGS & 11 & 5,39 & 6 & 2,94 & 18 & 8,82 & 6 & 2,94 & 20 & 9,80 & 61 & 29,90 \\
\hline UFSC & 4 & 1,96 & 10 & 4,90 & 3 & 1,47 & 8 & 3,92 & 10 & 4,90 & 35 & 17,16 \\
\hline UFSM & 4 & 1,96 & 6 & 2,94 & 14 & 6,86 & 2 & 0,98 & 6 & 2,94 & 32 & 15,69 \\
\hline Total & 40 & 19,61 & 42 & 20,59 & 45 & 22,06 & 30 & 14,71 & 47 & 23,04 & 204 & 100,00 \\
\hline
\end{tabular}


Tabela 3 - Distribuição das produções defendidas nos Programas de Pós-Graduação estudados, segundo tipo, que tiveram aderência aos eixos da ANPPS

\begin{tabular}{lcccccc}
\hline & \multicolumn{2}{c}{ Dissertação } & \multicolumn{2}{c}{ Tese } & \multicolumn{2}{c}{ Total } \\
& n & $\mathbf{\%}$ & $\mathbf{n}$ & $\mathbf{\%}$ & $\mathbf{n}$ & \% \\
\hline UFPel PPGO & 23 & 11,27 & 10 & 4,90 & 33 & 16,18 \\
UFPR PPGO & 43 & 21,08 & 0 & 0,00 & 43 & 21,08 \\
UFRGS PPGO & 34 & 16,67 & 27 & 13,24 & 61 & 29,90 \\
UFSC PPGO & 23 & 11,27 & 12 & 5,88 & 35 & 17,16 \\
UFSM PPCO & 32 & 15,69 & 0 & 0,00 & 32 & 15,69 \\
Total & 155 & 75,98 & 49 & 24,02 & 204 & 100,0 \\
\hline
\end{tabular}

Dos 17 eixos da ANPPS, 14 foram contemplados pela produção dos programas de pósgraduação, no período de estudo. Os eixos 3, 7 e 11 concentram, aproximadamente, $48 \%$ da produção (tabela 4).

A distribuição e nomenclatura das áreas de concentração dos programas de pós-graduação apresenta variabilidade entre as instituições (tabela 5). A UFSC possui o maior número de áreas de concentração, enquanto que a UFSM aglutina sua estrutura de pós-graduação em uma única grande área. As áreas de concentração mais disseminadas entre os programas e responsáveis por $44,60 \%$ de toda a produção aderida à ANPPS são Odontopediatria/SB durante a Infância e Adolescência, Saúde Coletiva e Diagnóstico Bucal/Estomatologia. As áreas de Odontopediatria e SB durante a Infância e Adolescência possuem a maior média de aderência à Agenda, entre as áreas de concentração $(16,00 \pm 11,13)$, seguidas pela Saúde Coletiva ou SB Coletiva $(14,33 \pm 5,03)$. As áreas de Materiais Dentários, Prótese Dentária e Endodontia detiveram as menores médias em números absolutos, representando $1,96 \%$ de teses ou dissertações aderidas à Agenda.

Tabela 4 - Distribuição da produção científica dos Programas de Pós-Graduação de acordo com os Eixos da ANPPS, segundo ano da defesa

\begin{tabular}{cccccccc}
\hline Eixos (ANPPS) & $\mathbf{2 0 1 1}$ & $\mathbf{2 0 1 2}$ & $\mathbf{2 0 1 3}$ & $\mathbf{2 0 1 4}$ & $\mathbf{2 0 1 5}$ & Total & \% \\
\hline 1 & 1 & 1 & 1 & 1 & 1 & 5 & 2,46 \\
2 & 0 & 2 & 0 & 1 & 3 & 6 & 2,95 \\
3 & 12 & 13 & 8 & 3 & 11 & 47 & 23,05 \\
4 & 1 & 4 & 7 & 0 & 6 & 18 & 8,82 \\
5 & 0 & 2 & 0 & 0 & 0 & 2 & 0,98 \\
6 & 4 & 3 & 1 & 4 & 3 & 15 & 7,35 \\
7 & 4 & 4 & 9 & 6 & 0 & 23 & 11,27 \\
8 & 1 & 2 & 3 & 2 & 1 & 9 & 4,41 \\
10 & 6 & 3 & 5 & 1 & 2 & 17 & 8,33 \\
11 & 7 & 4 & 5 & 7 & 6 & 29 & 14,21 \\
12 & 2 & 2 & 2 & 2 & 5 & 13 & 6,37 \\
14 & 2 & 2 & 2 & 1 & 3 & 10 & 4,90 \\
15 & 0 & 0 & 2 & 2 & 5 & 9 & 4,41 \\
16 & 0 & 0 & 0 & 0 & 1 & 1 & 0,49 \\
Total & 40 & 42 & 45 & 30 & 47 & 204 & 100,00 \\
\hline
\end{tabular}


Tabela 5 - Distribuição da produção científica dos Programas de Pós-Graduação segundo áreas de concentração, com adesão à ANPPS

\begin{tabular}{|c|c|c|}
\hline Programas e áreas de concentração & $\mathbf{n}$ & $\%$ \\
\hline UFPel PPGO & 33 & 16,18 \\
\hline Dentística & 13 & 6,37 \\
\hline Diagnóstico Bucal & 3 & 1,47 \\
\hline Materiais Dentários & 1 & 0,49 \\
\hline Odontopediatria & 14 & 6,86 \\
\hline Prótese Dentária & 2 & 0,98 \\
\hline UFPR PPGO & 43 & 21,08 \\
\hline Estomatologia & 6 & 2,94 \\
\hline $\begin{array}{l}\text { Saúde Bucal durante a Infância e } \\
\text { Adolescência }\end{array}$ & 28 & 13,73 \\
\hline Saúde Coletiva & 9 & 4,41 \\
\hline UFRGS PPGO & 61 & 29,90 \\
\hline Clínica Odontológica & 39 & 19,12 \\
\hline Patologia Bucal & 3 & 1,47 \\
\hline Saúde Bucal Coletiva & 19 & 9,31 \\
\hline UFSC PPGO & 35 & 17,16 \\
\hline Dentística & 2 & 0,98 \\
\hline Diagnóstico Bucal & 6 & 2,94 \\
\hline Endodontia & 1 & 0,49 \\
\hline Implantodontia & 5 & 2,45 \\
\hline Odontopediatria & 6 & 2,94 \\
\hline Saúde coletiva & 15 & 7,35 \\
\hline UFSM PPCO & 32 & 15,69 \\
\hline Odontologia & 32 & 15,69 \\
\hline Total & 204 & 100,00 \\
\hline
\end{tabular}

\section{DISCUSSÃO}

Neste estudo verifica-se que, apesar do crescimento contínuo da pós-graduação em Odontologia no país, a maior parte da produção de teses e dissertações analisadas não apresentou aderência à ANPSS (56,13\%). Além disto, maiores quantitativos de produção não significaram, necessariamente, maior proporção de aderência à Agenda. Verifica-se, também, uma desproporção na aderência aos eixos da Agenda: a UFRGS 
apresenta isoladamente a maior média de produção anual aderida, enquanto a UFPel e UFPR possuem a maior proporção média de aderência juntas. $\mathrm{O}$ que sugere um questionamento sobre o porquê desta diferença de adesão encontrada entre os programas de pós-graduação estudados e o real impacto da Agenda como política pública (PP).

Dados do estudo mostram as áreas de Odontopediatria e SB durante a Infância e Adolescência, como de maior média de aderência à Agenda entre as áreas de concentração, seguidas pela Saúde Coletiva ou Saúde Bucal Coletiva; e as áreas de Materiais Dentários, Prótese Dentária e Endodontia, como de menores médias de adesão à Agenda, refletindo uma fraca coordenação e integração entre as disciplinas para implementação prática da Agenda, com pouca visibilidade enquanto uma política central para a SB, incipiente diante da necessidade de resposta aos problemas sociais apontados.

$\mathrm{Na}$ formulação de novas PP, o Estado acaba desencadeando um conjunto de processos e relações, muitas vezes contraditórios, que mediam a formulação dessas políticas, criando novas oportunidades para determinados grupos e suprimindo outras, para outros ${ }^{16}$. No campo da política educacional, pode haver desacordos com o que se passa nos sistemas de ensino e nas escolas, porque "a política educacional como campo social é delimitada por atores alheios à educação - universidades, publicações, periódicos, pesquisadores (públicos e privados)" "15. Neste sentido, traçar um quadro mais adequado de efetivação prática da ANPPS, vislumbrando seus impactos e possibilidade de avanços, remete às tradicionais políticas de pesquisa em odontologia no Brasil.

A análise sobre a importância da implementação prática da Agenda, baseada na sua concretude, ou seja, no engajamento dos pesquisadores aos tópicos da Agenda, está para além da ne- cessária ampliação de sua visibilidade. A incipiente eficácia em sua implementação parece acontecer não por problemas de coerência interna no espaço da pesquisa em SB ou de coerência externa em acordo com as necessidades sociais apontadas; mas pela presença, ainda, de forte contraposição ao projeto da Agenda, marcadamente de uma tendência biotecnicista na ciência odontológica. O que perpassa, também, a maior ou menor possibilidade de publicação em periódicos científicos, uma exigência cada vez maior da vida acadêmica seguindo as orientações das agências de fomento de pesquisa brasileiras. A facilidade em uma posterior publicação acaba pesando na decisão sobre o tema a ser estudado. Considera-se: os temas de relevância social apontados pela ANPPS têm boa aceitação para publicação em periódicos nacionais e internacionais?

As PP tradicionais na Odontologia que deram forte abertura aos interesses privados em detrimento dos públicos, seguindo a linha de uma identidade e status profissional liberal, regulada pelo mercado e pelo complexo médico-industrial, ainda impulsionam substancialmente a busca pela "comunidade odontológica" de financiamento em agências de fomento ligadas às empresas (multinacionais), que desenvolvem tecnologia de ponta na área. Um paradigma de financiamento que se mostra socialmente descontextualizado e em consonância com as políticas neoliberais de Estado mínimo, prevalentes no Brasil até a década de 1990, mais presente em disciplinas consideradas eminentemente técnicas ou clínicas. Assim, parece possível apontar como agentes influentes na diferente adesão aos tópicos da Agenda, tanto a permanência das pesquisas que seguem o complexo médico-industrial e a pouca interdisciplinaridade existente entre as disciplinas, quanto o nível de adesão dos diferentes cursos às Diretrizes Curriculares Nacionais (DCN) ${ }^{17}$. 
Uma realidade de hegemonia histórica do complexo médico-industrial sobre as pesquisas em SB disputa com a nova política da ANPPS revelando um nó epistemológico ${ }^{18}$ entre $\mathrm{SB}$ e Odontologia, bem como os problemas na aplicação prática da Agenda. No caminho de reorganização da atenção primária e especializada, configura-se a tentativa de superação do histórico modelo liberal-biomédico por um novo modelo de trabalho multiprofissional e interdisciplinar, com ênfase na comunicação entre os atores e na integralidade da atenção à saúde. Um novo modus operandi que passa a ser assumido como estratégia de Estado, com base em mudanças qualitativas no âmbito do Estado brasileiro, introduzidas pelas políticas de humanização e a PNSB, que seguem uma nova lógica assistencial correlacionada às necessidades epidemiológicas e sociais. $\mathrm{Na}$ formação, as novas competências apontadas pelas DCN, passaram a ressaltar a importância do aluno cultivar uma nova relação de atenção, confiança, respeito e cuidado com o paciente/usuário e sua comunidade. O que aponta a necessidade de uma formação ética e (auto) crítica do futuro docente, além de reposicionar a importância do comprometimento da pesquisa científica em SB com as reais necessidades da população brasileira e do sistema de saúde.

A reordenação dos recursos humanos na saúde, construída em conjunto com políticas que englobam educação e trabalho, em uma intervenção deliberada para resolução de problemas sociais amplos e complexos, assumem caráter de PP transformadoras de valores ${ }^{19}$, onde o ensino e a pesquisa andam em compasso ao trabalho $^{20}$.

Um novo paradigma que impulsiona a implementação prática da ANPPS num contexto de disputa de valores ético-políticos, aflorando diferentes contradições: um sujeito epistêmico coletivo em contradição com um sujeito individual; um discurso integral do ser humano contraditório com um discurso ainda hegemônico da superioridade da hiperespecialização fragmentadora; um interesse público em contradição com interesses particularistas privados e de grupo; uma consciência profissional sobre os processos sociais que envolvem a SB dissociada de uma profissão ainda elitista e liberal; e uma pesquisa científica comprometida com os interesses / necessidades sociais contraposta a uma pesquisa subserviente aos interesses do complexo médicoindustrial.

No mesmo sentido, evidencia-se um insuficiente planejamento na distribuição das pesquisas nos cursos de graduação, de acordo com as necessidades sociais apontadas pela Agenda, ao configurar-se que dos 17 eixos, somente 14 foram contemplados pelas pesquisas, onde os eixos 3,7 e 11 concentram, aproximadamente, $48 \%$ desta produção, deixando os demais 14 eixos com baixa ou inexistente produção. Associadas a alterações no financiamento, mantém-se como desafio à odontologia na contemporaneidade, este comprometimento ético de priorização da pesquisa com a melhoria da qualidade de vida de ampla maioria dos brasileiros. No caminho da saúde percebida como direito e tendo como meta uma nova construção científica da odontologia, uma possível interdisciplinaridade e relação dialógica entre áreas clínicas e as disciplinas com sentido humano, ético e sanitarista, parece fazerse necessária. Entram em jogo novos projetos setoriais e societários a serem conquistados nesta interpelação entre a consolidação do SUS, avanços na formação voltados ao interdisciplinar e integral, e uma pesquisa comprometida com a qualidade dos serviços bucais prestados à ampla maioria da população brasileira - individuais ou coletivos.

Mudanças no sistema de avaliação de temas para publicação nos periódicos 
odontológicos brasileiros, a partir de uma relevância público-social e não apenas de mercado, precisam ser consideradas. $\mathrm{Na}$ atualidade é incomum um pesquisador, ao planejar um projeto de pesquisa com intuito de encaminhá-lo posteriormente à publicação, não levar em conta a classificação Qualis dos periódicos $^{21}$. Se há dificuldade em conseguir aprovação para publicação em revistas com alto impacto e bom Qualis-CAPES, de temas relacionados aos vários eixos propostos pela ANPPS, que prioriza estudos de relevância para o planejamento e gerenciamento de serviços em saúde, constrói-se um viés na escolha do tema de estudo que pode limitar a amplitude da produção científica.

\section{CONCLUSÃO}

Conclui-se que existem diferentes movimentos de assimilação e incorporação da Agenda na pesquisa em SB, com maior aderência nos cursos, disciplinas/áreas que incorporam um sentido sanitarista de coerência e articulação aos objetivos do Sistema de saúde e em consonância às DCN. A incorporação de uma mudança de direção e de prioridades dentro da lógica da Agenda, parece passar por um necessário salto interdisciplinar e de comprometimento ético-sanitarista, bem como por uma profunda reflexão sobre a priorização dos temas para publicação assumidos pelos periódicos científicos em odontologia, buscando facilitar a publicação dos estudos compatíveis com os eixos da ANPPs, por sua relevância social. No mesmo sentido, solicita-se inovação conceitual das disciplinas para suplantar a mera reprodução do conhecimento e da pesquisa de ponta desenvolvidos nos países centrais da economia mundial, percebendo-se, assim, como parte importante da pesquisa voltada ao desenvolvimento do Brasil. A incorporação desta Agenda em pesquisa, pautada nas necessidades impostas pela realidade social, pode alçar a
Odontologia brasileira à referência, não só para transformação da realidade do país, mas das nações latino-americanas. O que remete à necessidade de constante (auto) avaliação dos itens da Agenda em consonância à sua aplicação prática, para manutenção de sua coerência.

\section{ABSTRACT \\ The scientific production of Dentistry and the National Agenda for Priorities in Health Re- search}

The study analyzed the current production of oral health scientific knowledge in Southern Brazil dentistry stricto-sensu post-graduation programs and the relation with the National Agenda for Priorities in Health Research guidelines. It is an exploratory-descriptive research, with a quantitative-qualitative approach, based on documentary analysis. The data, collected from the post-graduation electronic sites in 2015, consisted of theses and dissertations produced between 2011 and 2015 , by the "Dentistry" or "Dental Sciences" programs of Federal Universities of Southern Brazil. After reading the titles and abstracts, the productions were classified according to the Agenda axes, with the subsequent organization, tabulation, quantitative and qualitative analysis. It was discussed possible lapses between public policies and practices, in the implementation of the Agenda. Most of the theses and dissertations analyzed did not show adherence to the Agenda axes, with Pediatric Dentistry and Public Health areas having the highest associated production. Although there are movements of incorporation of the Agenda in oral health research, traditional clinical disciplines still are not used to refer it or to focus on the population needs and the Health System interest. The incorporation of the Agenda into dental research can possibly transform Brazilian dentistry in a new reference of construction of knowledge beyond the national context.

Descriptors: Oral Health. Dentistry. PostGraduate Education in Dentistry.

\section{REFERÊNCIAS}

1. Cury JA. The dilemma of researchers, the insensibility of policy-makers and the distress 
of Brazilian dentistry journals. Braz Oral Res. 2012;26 (2):92.

2. Garrafa V, Moysés SJ. Odontologia brasileira: tecnicamente elogiável, cientificamente discutível, socialmente caótica. Divulg Saúde Debate. 1996;(13):617.

3. Sigolo BOO, Casarin HCS. Destaque da Produção Científica Brasileira em Odontologia no cenário mundial e a influência no comportamento informacional do profissional cirurgião-dentista. Rev EDICIC. 2011;1(4):389-407.

4. Gracio MCC, Oliveira EFT, Gurgel JA, Escalona MI, Guerrero AP. Dentistry scientometric analysis: a comparative study between Brazil and other most productive countries in the area. Scientometrics. 2013; 95(2): 753-69.

5. Scimago Journal Rank Indicator. [Acesso em 20 jun. 2016]. Disponível em: http://www. scimagojr.com/index.php.

6. Normando D. A ciência odontológica brasileira. Dent Press J Orthod. 2014;19(2) $: 14$.

7. Souza JGS, Popoff DAV, Oliveira RCN, Almeida ER, Martelli Junior H, Lima AMEB. Profile and scientific production of Brazilian researchers in dentistry. Arq Odontol. 2016 :52(1):13-22.

8. Dias AA, Narvai PC, Rego DM. Tendências da produção científica em odontologia no Brasil. Rev Panam Salud Publica. 2008; 24(1):54-60.

9. Brasil. Ministério da Saúde. SB Brasil Pesquisa Nacional de Saúde Bucal 2010: Resultados Principais. Brasília: Ministério da Saúde; 2011. [Acesso em 10 jan. 2017.] Disponível em: http://189.28.128.100/dab/ docs/portaldab/publicacoes/pesquisa_saude bucal.pdf.

10. Moysés SJ. O contexto atual para a pesquisa em Saúde Bucal Coletiva. Cad Saúde Pública. 2008; 24(4):718-9.

11. Novaes HMD. Tecnologias e saúde: a construção social da prática odontológica. In: Botazzo C, Freitas SFT. (Orgs.). Ciências sociais e saúde bucal. São Paulo: UNESP/EDUSC, 1998. p.141-58.

12. Frazão P. Tecnologias em saúde bucal coletiva. In: Botazzo C, Freitas SFT. (Orgs.). Ciências sociais e saúde bucal. São Paulo: UNESP/EDUSC; 1998.p.159-74.

13. Brasil. Ministério da Saúde. Brasil Sorridente. Portal do Departamenteo de Atenção Básica (atenção hospitalar). [Acesso em 10 jan. 2017.] Disponível em: http://189.28.128.100/dab/docs/publicacoes /geral/diretrizes_da_politica_nacional_de_saude_bucal.pdf.

14. Brasil. Ministério da Saúde. Agenda Nacional de Prioridades de Pesquisa em Saúde. Brasília: Ministério da Saúde; 2011. [Acesso em 10 jan. 2017.] Disponível em: http://bvsms.saude.gov.br/bvs/publicaoes/a genda_nacional_prioridades_2ed_3imp.pdf.

15. Blackmore J, Hugh L. Pesquisa de Políticas. In: Somekh B, Lewin C. (Orgs.). Teoria e Métodos de Pesquisa Social. Rio de Janeiro: Vozes; 2015. p.253-61.

16. Cejudo GM, Michel CL. Coherencia y políticas públicas: metas, instrumentos y poblaciones objetivo. Gestión y Política Pública 2016; 25:3-31.

17. Brasil. Ministério da Educação. Diretrizes Curriculares Nacionais. [Acesso em 8 jul. 2015]. Disponível em: http://portal.mec. gov.br/index.php?option $=$ com content $\&$ id $=$ 12991:diretrizes-curriculares-cursos-de-gra duacao.

18. Emmerich A, Castiel LD. A ciência odontológica, Sísifo e o "efeito camaleão". Interface Comun Saúde Educ. 2009; 13(29):339-51. 
19. Freitas AF, Freitas AF, Ferreira MAM. Gestão social como projeto político e prática discursiva. Cad. EBAPE.BR 2016; 14(2):Art. 3.

20. Gaztanaja J. La política del valor y la política del significado: tendiendo puentes. Antipod Rev Antropol Arqueol. 2016; 24:111-30.

21. Trzesniak P. As dimensões da qualidade dos periódicos científicos e sua presença em um instrumento da área da educação. Rev Bras Educ. 2006; 11 (32): 346-377.

Correspondência para:

Doris Gomes

e-mail: dorisgomesodonto@gmail.com

Rod. Rafael da Rocha Pires, 3913, Sambaqui, 88051-001 - Florianópolis/SC 